\title{
artigo
}

Taveira, L.M.; Aguiar, R.S.

Mortalidade por suicídio na população idosa do distrito federal, 2010 a 2019

\section{Mortalidade por suicídio na população idosa do distrito federal, 2010 a 2019}

\author{
Suicide mortality in the elderly population of the federal district, 2010 to 2019 \\ Mortalidad por suicidio en la población mayor del distrito federal, 2010 a 2019
}

\section{RESUMO}

Objetivo: Caracterizar casos de mortalidade por suicídio no período de 2010-2019 em pessoas idosas no Distrito Federal. Método: Trata-se de um estudo descritivo, retrospectivo, exploratório com um recorte transversal de dados secundários. A amostra foi obtida por meio da disponibilização de dados do Sistema de Informação sobre Mortalidade. Os dados foram organizados em planilha do Excel e a análise se deu mediante o emprego de estatística descritiva. Resultados: Identificou-se a ocorrência de 165 óbitos por suicídio entre idosos no período. Os óbitos estiveram mais presentes nos homens, na faixa de idade de 60 a 69 anos, em casados, em pessoas com pouco estudo e em pardos. 0 enforcamento, estrangulamento e sufocação foi o modo mais adotado, tendo o domicílio como o principal local de ocorrência. Conclusão: Torna-se necessário adotar estratégias para que medidas preventivas ao suicídio em idosos sejam implementadas pelos profissionais de saúde de modo a mitigar novas ocorrências. DESCRITORES: Mortalidade; Suicídio; Idoso.

\section{ABSTRACT}

Objective: To characterize cases of mortality by suicide in the period 2010-2019 in old people in the Federal District. Method: This is a descriptive, retrospective, exploratory study with a cross section of secondary data. The sample was obtained through the availability of data from the Mortality Information System. The data were organized in an Excel spreadsheet and the analysis was performed using descriptive statistics. Results: It was identified the occurrence of 165 deaths by suicide among elderly people in the period. Deaths were more prevalent in men, aged 60 to 69 years, married, in people with little education and browns. Hanging, strangulation and suffocation was the most adopted method, with the home as the main place. Conclusion: It becomes necessary to adopt strategies so that preventive measures for suicide in the elderly are implemented by health professionals in order to mitigate new occurrences.

DESCRIPTORS: Mortality; Suicide; Aged.

\section{RESUMEN}

Objetivo: Caracterizar casos de mortalidad por suicidio en el período 2010-2019 en personas mayores en el Distrito Federal. Método: Se trata de un estudio exploratorio, descriptivo, retrospectivo, con un corte transversal de datos secundarios. La muestra se obtuvo mediante la disponibilidad de datos del Sistema de Información de Mortalidad. Los datos se organizaron en una hoja de cálculo Excel y el análisis se realizó mediante estadística descriptiva. Resultados: Se identificó la ocurrencia de 165 muertes por suicidio entre ancianos en el período. Las muertes fueron más prevalentes en hombres, de 60 a 69 años, casados, en personas con poca educación y morenos. El ahorcamiento, estrangulamiento y asfixia fue el método más adoptado, siendo el hogar el principal lugar. Conclusión: se hace necesario adoptar estrategias para que los profesionales de la salud implementen las medidas preventivas del suicidio en el adulto mayor con el fin de mitigar nuevos episódios.

DESCRIPTORES: Mortalidad; Suicidio; Anciano.

RECEBIDO EM: 16/05/2021 APROVADO EM: 28/05/2021

\section{Lúcia de Medeiros Taveira}

Universidade Paulista (UNIP), Campus Brasília, Distrito Federal, Brasil. Curso de Enfermagem.

ORCID: 0000-0001-9907-2183

\section{Ricardo Saraiva Aguiar}

Universidade Paulista (UNIP), Campus Brasília, Distrito Federal. Curso de Enfermagem.

ORCID: 0000-0003-0335-2194 


\section{INTRODUÇÃO}

0 suicídio é definido como um ato intencional de matar a si mesmo ${ }^{1} \mathrm{e}$ está presente em diferentes idades, raças e classes sociais. Apresenta-se como um fenômeno social, sendo ainda um tabu devido a grande ferida emocional que poucos conseguem aceitar e/ou falar. ${ }^{2}$

Para a Organização Mundial da Saúde (OMS), os números relacionados ao suicídio não devem ser ignorados e, muito provavelmente, são maiores que os disponíveis nas notificações, pois há ainda uma evidente subnotificação de casos pelos serviços de saúde e órgãos de segurança pública. Mesmo nos países com bons dados de registro, os episódios podem estar sendo classificados como outro tipo de morte. ${ }^{3}$

Tratado com estigma social e também com muitos tabus, o suicídio ocorre em todo o mundo e tem sido tratado como um problema de saúde pública. De acordo com a OMS, estima-se um percentual de que $75 \%$ dos suicídios ocorra em países de baixa renda. ${ }^{4}$

Meios comuns como a ingestão de inseticidas, enforcamento e até mesmo o uso armas de fogo são as causas mais frequentes de suicídio. Conflitos sociais que abrange desde guerras e discriminações de todas as formas e gêneros, têm aumentado substancialmente as tentativas de suicídio, bem como de suicídio evidenciado. Nota-se também que problemas de saúde mental como a depressão e a ingestão abusiva de álcool têm presença marcante em países de renda mais elevada, além de crises que afetam o poderio econômico. ${ }^{4}$ Ademais, com o envelhecimento da população, tem-se notado aumento nos casos de suicídio na população idosa. ${ }^{5}$

No processo de envelhecimento, constata-se a vivência de situações altamente desvitalizantes, com frequente comprometimento subjacente da saúde mental, podendo conduzir a quadros depressivos que, por muitas vezes, pode determinar a ocorrência de pensamentos suicidas e/ou a execução do ato. Acredita-se que dois terços dos suicídios na população idosa estão relacionados à depressão. ${ }^{5-7}$
Para a Organização

Mundial da Saúde

(OMS), os números

relacionados

ao suicídio

não devem ser

ignorados e, muito

provavelmente,

são maiores que

os disponíveis nas

notificações, pois há

ainda uma evidente

subnotificação de

casos pelos serviços

de saúde e órgãos de

segurança pública.

Mesmo nos países

com bons dados de

registro, os episódios

podem estar sendo

classificados como

outro tipo de morte.
Projeções populacionais da Organização das Nações Unidas (ONU) indica que até o ano de 2050, o número mundial de pessoas idosas poderá atingir cerca de 2,1 bilhões. No Brasil, a porcentagem de pessoas idosas tende a aumentar de $12 \%$ em 2015 para 29\% em 2050. Assim, esse processo de transição sociodemográfica requer políticas públicas para garantir o envelhecimento ativo e saudável, inclusive que aborde a prevenção do suicídio. ${ }^{8-9}$

O Ministério da Saúde, através da Portaria $\mathrm{n}^{\circ} 1.876$, de 14 de agosto de 2006, instituiu as diretrizes nacionais para prevenção do suicídio com ações de cuidado integral através de medidas de promoção, prevenção, tratamento e recuperação da saúde, bem como a identificação de determinantes e condicionantes do suicídio e/ou tentativas, além da capacitação dos profissionais da atenção primária à saúde (APS) para o adequado conhecimento e manejo dos fatores envolvidos. ${ }^{10}$

Assim, diante do evidente crescimento populacional de idosos, torna-se essencial o reconhecimento e o preparo para as demandas pessoais e coletivas relacionadas aos aspectos biológicos, psicológicos $\mathrm{e}$ socioambientais que advêm com a idade. Este assunto é de grande importância, pois traz a discussão sobre um tema cercado de tabus e preconceitos, historicamente incômodo para a sociedade e para governantes de todo o mundo, o suicídio.

Diante da complexidade e magnitude das questões que envolvem o suicídio na população idosa, este estudo tem o objetivo de caracterizar os casos de mortalidade por suicídio no período de 2010-2019 em pessoas com idade igual ou superior a 60 anos no Distrito Federal.

\section{MÉTODOS}

Trata-se de um estudo descritivo, retrospectivo, exploratório com um recorte transversal de dados secundários sobre a mortalidade por suicídio na população idosa, por região administrativa do Distrito Federal, no período de 2010-2019.

A amostra foi obtida por meio da disponibilização de informações pela $\mathrm{Se}$ - 


\section{artigo}

Taveira, L.M.; Aguiar, R.S.

Mortalidade por suicídio na população idosa do distrito federal, 2010 a 2019

cretaria de Estado da Saúde do Distrito Federal (SES/DF) através do Sistema de Informação sobre Mortalidade (SIM). Foram considerados como suicídio os óbitos por lesões autoprovocadas voluntariamente de acordo com $10^{a}$ revisão da Classificação Internacional de Doenças (CID10), a saber: X60 a X84.

\section{Tabela 1 - Distribuição dos óbitos por suicídio e totais no período de 2010- \\ 2019 em pessoas com idade igual ou superior a 60 anos no Distrito Federal, Brasil, segundo o ano de ocorrência.}

\begin{tabular}{|c|c|c|c|c|c|c|}
\hline \multirow{2}{*}{\multicolumn{2}{|c|}{ VARIÁVEL }} & \multicolumn{2}{|c|}{ ÓBITOS POR SUICIIDIO } & \multicolumn{2}{|c|}{ ÓBITOS TOTAIS } & \multirow[t]{2}{*}{ PREVALÊNCIA } \\
\hline & & $\mathbf{N}$ & $\%$ & $\mathbf{N}$ & $\%$ & \\
\hline \multirow[t]{10}{*}{ Ano } & 2010 & 18 & 10,9 & 5871 & 8,4 & 0,31 \\
\hline & 2011 & 10 & 6,1 & 6107 & 8,7 & 0,16 \\
\hline & 2012 & 19 & 11,5 & 6212 & 8,9 & 0,31 \\
\hline & 2013 & 10 & 6,1 & 6429 & 9,2 & 0,16 \\
\hline & 2014 & 10 & 6,1 & 6982 & 10,0 & 0,14 \\
\hline & 2015 & 15 & 9,1 & 7119 & 10,2 & 0,21 \\
\hline & 2016 & 16 & 9,7 & 7214 & 10,3 & 0,22 \\
\hline & 2017 & 15 & 9,1 & 1865 & 11,3 & 0,19 \\
\hline & 2018 & 27 & 16,4 & 7694 & 11,0 & 0,35 \\
\hline & 2019 & 25 & 15,2 & 8354 & 12,0 & 0,30 \\
\hline \multicolumn{2}{|c|}{ TOTAL } & 165 & 100 & 69847 & 100 & 0,23 \\
\hline
\end{tabular}

Tabela 2 - Distribuição dos óbitos por suicídio no período de 2010-2019 em pessoas com idade igual ou superior a 60 anos no Distrito Federal, Brasil, segundo o sexo, faixa etária, estado civil, grau de instrução, raça/cor e local de ocorrência.

$\begin{array}{llcc}\text { VARIÁVEL } & & \mathbf{N} & \% \\ \text { Sexo } & \text { Masculino } & 124 & 75,2 \\ & \text { Total } & 41 & 24,8 \\ \text { Faixa etária } & 60-69 \text { anos } & 165 & 100 \\ & 70-79 \text { anos } & 95 & 57,6 \\ & 80-89 \text { anos } & 52 & 31,5 \\ & 90 \text { anos ou mais } & 15 & 9,1 \\ & \text { Total } & 3 & 1,8 \\ \text { Estado Civil } & \text { Solteiro } & 165 & 100 \\ & \text { Casado } & 33 & 20,0 \\ & \text { Viúvo } & 77 & 46,7 \\ & \text { Separado judicialmente } & 18 & 10,9 \\ & \text { União consensual } & 34 & 20,6 \\ & \text { Total } & 3 & 1,8\end{array}$

com idade igual ou superior a 60 anos que tivessem cometido suicídio no período de 1 de janeiro de 2010 a 31 de dezembro de 2019. Foram excluídos os óbitos por suicídio de pessoas com idade inferior a 60 anos ou fora do período de 1 de janeiro de 2010 a 31 de dezembro de 2019.

Considerou-se 165 casos de óbitos por suicídio de idosos no Distrito Federal. Os dados foram organizados em planilha do Excel e a análise se deu mediante o emprego de estatística descritiva, organizando os dados numéricos em tabelas de distribuição de frequência absoluta e percentuais.

Este estudo atendeu aos preceitos éticos da Resolução do Conselho Nacional de Saúde (CNS) n ${ }^{\circ} 510$, de 07 de abril de 2016. Por utilizar apenas dados sem identificação dos sujeitos, foi dispensada a submissão ao Comitê de Ética em Pesquisa (CEP).

\section{RESULTADOS}

No período de 2010 a 2019, ocorreram 165 óbitos por suicídio nas pessoas com faixa etária de 60 anos ou mais no Distrito Federal. Assim, percebe-se maior número de casos no ano de 2018 com 27 óbitos (16,4\%), seguido de 2019 com 25 (15,2\%) e 2012 com 19 (11,5\%) (Tabela 1).

Quanto à prevalência dos óbitos, identifica-se maior prevalência no ano de 2018 com $0,35 \%$, seguido de 2010 e 2012 com $0,31 \%$ (cada ano) e 2019 com $0,30 \%$, conforme Tabela 1 a seguir.

A tabela 2 apresenta a distribuição dos óbitos segundo o sexo, faixa etária, estado civil, grau de instrução, raça/cor e local de ocorrência. Diante disso, os episódios de suicídio foram mais presentes nos homens (124 óbitos - 75,2\%), nas pessoas com faixa de idade de 60 a 69 anos (95 casos - 57,6\%), em casados (77 casos - 46,7\%), que tinham de 4 a 7 anos de estudo ( $46 \mathrm{ca}$ sos $-27,9 \%)$, eram da raça/cor classificada como pardas ( 85 casos $-51,5 \%$ ) e tiveram como local de ocorrência do óbito o domicílio ( 104 casos - 63\%).

Sobre a região administrativa do Distrito Federal de residência dos idosos com 60 anos ou mais que evoluíram ao óbito 


\begin{tabular}{|lccc}
\hline & 8-11 anos & 32 & 19,4 \\
& 12 ou mais & 44 & 26,7 \\
& Não informado & 2 & 1,2 \\
& Ignorado & 2 & 1,2 \\
& Total & 165 & 100 \\
& Branca & 75 & 45,5 \\
& Preta & 4 & 2,4 \\
Raça/Cor & Amarela & 0 & 0 \\
& Parda & 85 & 51,5 \\
& Indígena & 1 & 0,6 \\
& Total & 165 & 100 \\
& Hospital & 33 & 20,0 \\
& Outro Estabelecimento de Saúde & 2 & 1,2 \\
Local de Ocorrência & Domicilio & 104 & 63,0 \\
& Via pública & 11 & 6,7 \\
& Outros & 15 & 9,1 \\
& Total & 165 & 100 \\
& Fonte: Sistema de Informação sobre Mortalidade (2020). & &
\end{tabular}

Tabela 3 - Distribuição dos óbitos por suicídio no período de 2010-2019 em pessoas com idade igual ou superior a 60 anos no Distrito Federal, Brasil, segundo a Região Administrativa de residência.

\begin{tabular}{|c|c|c|c|}
\hline VARIÁVEL & & $\mathbf{N}$ & $\%$ \\
\hline & Paranoá & 1 & 0,6 \\
\hline & Núcleo Bandeirante & 1 & 0,6 \\
\hline & Itapoã & 1 & 0,6 \\
\hline & Cruzeiro & 2 & 1,2 \\
\hline & Riacho Fundo II & 2 & 1,2 \\
\hline & Park Way & 2 & 1,2 \\
\hline & São Sebastião & 3 & 1,8 \\
\hline & Riacho Fundo & 3 & 1,8 \\
\hline & Recanto das Emas & 4 & 2,4 \\
\hline Rogiān Adminictrativa & Lago Norte & 4 & 2,4 \\
\hline 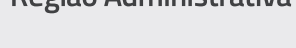 & Sobradinho II & 4 & 2,4 \\
\hline & Brazlândia & 5 & 3,0 \\
\hline & Sobradinho & 5 & 3,0 \\
\hline & Guará & 5 & 3,0 \\
\hline & Santa Maria & 5 & 3,0 \\
\hline & Sudoeste/Octogonal & 5 & 3,0 \\
\hline & Gama & 6 & 3,6 \\
\hline & Lago Sul & 6 & 3,6 \\
\hline & Vicente Pires & 7 & 4,2 \\
\hline & Planaltina & 10 & 6,1 \\
\hline
\end{tabular}

em decorrência do suicídio, a tabela 3 traz o Plano Piloto com $12,7 \%$ dos casos (21 óbitos), seguido de Águas Claras, Ceilândia e Taguatinga com $10,3 \%$ em cada uma (17 casos em cada região administrativa).

No que se refere às causas dos óbitos, as lesões autoprovocadas intencionalmente por enforcamento, estrangulamento e sufocação foram mais prevalentes com 82 óbitos (49,7\%), seguido pelas lesões autoprovocadas intencionalmente por precipitação de um lugar elevado com 19 óbitos (11,5\%) e pelas lesões autoprovocadas intencionalmente por disparo de arma de fogo com 12 óbitos (7,3\%), conforme apresentado na Tabela 4 a seguir.

\section{DISCUSSÃO}

Este estudo abordou sobre a mortalidade em idosos no Distrito Federal em decorrência do suicídio. Observa-se no período oscilações nos índices de mortalidade. Em 2010 foi registrado 18 mortes, mas em 2018 e 2019 registraram-se, respectivamente, 27 e 25 óbitos. Esta situação corrobora com a literatura que apresenta taxas de suicídio cada vez maior nos idosos, sendo necessário o avanço das pesquisas $\mathrm{e}$ realização de ações que tenham como objetivo a minimização da ocorrência desse fenômeno. ${ }^{1}$

No que diz respeito ao modo como os idosos vem praticando o suicídio, tal pesquisa se alinha a outras já realizadas que identificou o uso de métodos que sejam letais e rápidos ${ }^{12}$, fato que explica os altos índices de mortes no Distrito Federal por lesões autoprovocadas intencionalmente por enforcamento, estrangulamento e sufocação (82 óbitos - 49,7\%), lesões autoprovocadas intencionalmente por precipitação de um lugar elevado (19 óbitos - 11,5\%) e lesões autoprovocadas intencionalmente por disparo de arma de fogo (12 óbitos - 7,3\%). Tais maneiras de suicídio também são consideradas mais viáveis pelos idosos, já que não precisam de tanto esforço e técnica para execução. ${ }^{13}$

Permanecer por muito tempo sozinho ou ainda por achar que não são mais úteis à sociedade, estando cada vez mais dentro 


\section{artigo}

Taveira, L.M.; Aguiar, R.S.

Mortalidade por suicídio na população idosa do distrito federal, 2010 a 2019

$\begin{array}{llcc} & \text { Samambaia } & 12 & 7,3 \\ & \text { Taguatinga } & 17 & 10,3 \\ & \text { Ceilândia } & 17 & 10,3 \\ \text { Região Administrativa } & \text { Águas Claras } & 17 & 10,3 \\ & \text { Plano Piloto } & 21 & 12,7 \\ & \text { Total } & 165 & 100\end{array}$

Tabela 4 - Distribuição dos óbitos por suicídio no período de 2010-2019 em pessoas com idade igual ou superior a 60 anos no Distrito Federal, Brasil, segundo a causa.

VARIÁVEL

X61 - Auto-intoxicação por exposição intencional a anticonvulsivantes, sedativos, hipnóticos, antiparkinsonianos e psicóticos

X62 - Auto-intoxicação por exposição intencional a narcóticos e psicodislépticos

X64 - Auto-intoxicação por exposição intencional a outras drogas, medicamentos e substâncias biológicas

X65 - Auto-intoxicação voluntária por álcool

X68 - Auto-intoxicação por exposição intencional a pesticidas

X69 - Auto-intoxicação por exposição intencional a outros produtos químicos e substâncias nocivas

X70 - Lesão autoprovocada intencionalmente por enforcamento, estrangulamento e sufocação

X71 - Lesão autoprovocada intencionalmente por afogamento submersão

X72 - Lesão autoprovocada intencionalmente por disparo de

Causa do

Óbito arma de fogo

X73 - Lesão autoprovocada intencionalmente por disparo de espingarda, carabina ou arma de fogo de maior calibre

X74 - Lesão autoprovocada intencionalmente por disparo de outra arma de fogo e de arma de fogo não especificada

X76 - Lesão autoprovocada intencionalmente pela fumaça, pelo fogo e por chamas

X78 - Lesão autoprovocada intencionalmente por objeto cortante ou penetrante

X79 - Lesão autoprovocada intencionalmente por objeto contundente

X80 - Lesão autoprovocada intencionalmente por precipitação de um lugar elevado

X84 - Lesão autoprovocada intencionalmente por meios não especificados

Total

Fonte: Sistema de Informação sobre Mortalidade (2020).

de um ambiente caracterizado como residencial, seja a própria casa ou ainda em Instituições de Longa Permanência para
Idosos (ILPI), são fatores apresentados na literatura que podem potencializar os idosos a apresentarem ideações suicidas ou até mesmo cometerem suicídio dentro do próprio ambiente domicíliar. ${ }^{14}$

Ainda na atualidade, é comum observar a falta de estudos dentre os idosos que cometeram suicídio. Parte dos idosos analisados nessa pesquisa nasceram na década de 60 ou antes disso, época em que era muito comum homens e mulheres trabalharem em casas de família ou fazendas, não obtendo de tempo ou condição financeira para cuidar da própria saúde ou de sua instrução pessoal, o que acarretava, ao longo dos anos, no acúmulo de comorbidades e doenças crônicas não transmissíveis, e posteriormente na anulação dos mesmos do mercado de trabalho e para suas famílias, quando tinham. ${ }^{14} \mathrm{Tal}$ informação corrobora com os resultados desta pesquisa que identificou pouca instrução educacional entre os idosos que cometeram o autoextermínio.

A instrução educacional antigamente era um benefício para poucos. Devido a isso, condições e jornadas de trabalho duras e árduas eram vivenciadas pela população geral na busca pelo sustento próprio e de suas famílias, o que causava desgaste físico, emocional, invalidez e descarte. ${ }^{14}$

O gênero masculino, como mostra nessa pesquisa, foi o mais afetado por suicídio (124 óbitos - 75,2\%). Por tratar-se de uma figura considerada autônoma, imponente, viril, forte e corajosa e que não demonstra dor ou resistência aos esforços, pode levar a estes homens a pensamentos autodestrutivos quando este é descartado ou anulado por seus empregadores ou por sua família. Sendo assim, o gênero é considerado como um fator de vulnerabilidade, corroborando com outros estudos realizados. ${ }^{12,15}$

Outro fator comum e relevante na prevalência de suicídio em idosos é a perda do parceiro ou ainda problemas conjugais. ${ }^{5}$ Em 77 dos casos (46,7\%), notou-se a presença do matrimônio. Outro estudo realizado no ano de 2020 corroborou com este resultado quando trouxe relatos de que, parte dos idosos, quando são casados, principalmente ao descobrir alguma doença, tentam por vezes chamar a atenção do seu parceiro, ser visto e ser escutado afim de compartilhar do seu problema, o que 
nem sempre é reconhecido ou entendido. Tal sentimento pode despertar neles desespero e medo do que possa acontecer e mais ainda da dependência de um outro alguém para lhe prestar um cuidado na velhice. Dessa forma, o casamento também passa a englobar um dos fatores de vulnerabilidade para o aumento de autoextermínio em pessoas idosas. ${ }^{15-16}$

Em relação ao local de maior ocorrência dos óbitos, nota-se que os mesmos estão presentes, em maior porcentagem, na região administrativa do Plano Piloto $(12,7 \%)$ no Distrito Federal. Esta região administrativa apresenta população com maior poder aquisitivo, o que ressalta o fato de que a situação financeira nem sempre pode estar relacionada a felicidade. ${ }^{16}$

Outros estudos trouxeram relatos dessa mesma questão, onde é notório que, mesmo em famílias bem sucedidas financeiramente e dispondo de todo o conforto possível para a velhice, parte dos idosos sentem-se sozinhos e carentes afetivamente, imprimindo comentários e sentimentos sobre desamor por parte de filhos c demais familiares, além de maus tratos físicos e verbais, passando a sentirem-se como vítimas em um cenário que era para ser de acalento. ${ }^{1416}$

Cabe ressaltar que, apesar de a região administrativa do Plano Piloto ter uma parcela de vulnerabilidade ao suicídio em idosos, ela não é um fator predominante, tendo em vista que regiões como Águas Claras, que é considerada moradia de pessoas classificadas como média e alta renda e Taguatinga e Ceilândia, consideradas moradia de classe baixa e média, contam com mesmo percentual de óbitos (10,3\%), correspondendo a 17 óbitos por região administrativa. Neste caso, o fator predominante pode estar no próprio convívio com a família e não necessariamente na região administrativa de residência do idoso ou ainda pela dificuldade de acesso aos serviços de saúde locais. ${ }^{16}$

Falas e expressões de sentimentos de tristeza por parte dos idosos que cometeram suicídio, na maioria das vezes não são levadas a sério, o que acarreta em discussões familiares, com a verbalização de pala- vras impensadas que culminam em pensamentos autodestrutivos efetivados dentro do domicílio pelo próprio idoso. ${ }^{14,16}$

\section{Em relação ao}

local de maior

ocorrência dos

óbitos, nota-se que

os mesmos estão

presentes, em maior

porcentagem, na

região administrativa

do Plano Piloto

$(12,7 \%)$ no Distrito

\section{Federal.}

Temas como suicídio já são bastante debatidos. No entanto, por não haver uma notificação de tentativa de autoextermínio, a complexidade desse assunto pode não ser tratada da forma devida, o que inviabiliza o planejamento e a implementação de estratégias que minimizem a ocorrência desse fenômeno. As estatísticas têm apontado um destaque para o perfil idoso, o que pode ser justificado pela falta de um atendimento individualizado e personalizado para esse público. É de extrema importância, que, principalmente as Unidades Básicas de Saúde (UBS) estejam aptas e com profissionais capacitados para a identificação $\mathrm{c}$ acolhimento de indivíduos que manifestam pensamentos ou tenham intenções autodestrutivas. ${ }^{17}$

Toda a equipe de saúde, bem como a família que presta cuidado à pessoa idosa deve estar atenta a possíveis fatores que venham a contribuir para a tentativa e/ ou ocorrência de suicídio. A descoberta de doença incurável ou ainda o estágio terminal de alguma comorbidade pode conferir ao idoso o sentimento de medo e de incompetência com a própria vida. Nesses casos, faz-se necessário um aconselhamento diário, atividades laborais para ocupar a mente e desviar pensamentos incomuns. ${ }^{17}$

Evitar a facilidade ao suicídio do idoso deve ser uma questão pensada em grupo. Cabe ressaltar que a atividade em equipe é que vai garantir a ele a recuperação da saúde mental, evitando complicações e agravos através de uma promoção da saúde segura e eficaz, ou seja, orientar não somente o usuário, mas também àqueles que o cercam, seja em seu ambiente domiciliar, de lazer ou ocupacional. Prevenir que medicamentos controlados, armas de fogo e produtos agrotóxicos como inseticidas e pesticidas fiquem sobre o domínio dos mesmos em momentos de crises depressivas e perturbações mentais é essencial para evitar tal acidente. ${ }^{17-18}$

Dessa forma, os declínios de casos de suicídio nos anos de 2011, 2013 e 2014 no Distrito Federal podem estar relacionados às medidas implementadas e adotadas pela saúde e pela mídia a respeito do tema. No entanto, o aumento pode estar associado a vertente que tais práticas caem em desuso quando os índices de suicídio abaixam ${ }^{7,10}$, o que explica um aumento considerável de 2015 a 2017 e um aumento excessivo nos anos de 2018 (27 óbitos), o maior de todos os anos, e 2019 (25 óbitos).

Assim, é evidente que essa falha precisa ser revisada afim de que, cada vez mais, os profissionais de saúde e as políticas públicas tenham ênfase no que diz respeito à promoção da saúde mental do idoso para que se evite as tentativas de suicídio c o desfecho final que é o óbito. ${ }^{17-19}$ Falar sobre suicídio torna-se relevante e atual, configurando a ser um problema de saúde pública que requer uma maior atenção e criação de estratégias para reduzir a sua incidência. Portarias, recomendações e leis são criadas, mas torna-se necessário ações integradas nas diferentes esferas sócio psíquicas que envolvam toda a sociedade. ${ }^{20}$ 


\section{artigo}

Taveira, L.M.; Aguiar, R.S.

Mortalidade por suicídio na população idosa do distrito federal, 2010 a 2019

Portanto, é socialmente relevante investigar fatores relacionados ao suicídio em idosos com o intuito de prevenir a sua ocorrência e favorecer o bem-estar dos idosos porque nessas situações está em jogo a dignidade do ser humano, objetivada na qualidade de vida dos envolvidos. Do ponto de vista do progresso e das funções das instituições sociais e de saúde, importa criar condições que promovam o ser humano independentemente de sua idade, condição social, sexo e profissão. ${ }^{21-22}$

\section{CONCLUSÃO}

Os resultados deste estudo revelaram a ocorrência de 165 óbitos por suicídio entre idosos do Distrito Federal no período, tendo maior prevalência no ano de 2018. Além disso, esteve mais presente nos homens, nas pessoas com faixa de idade de 60 a 69 anos, em casados, em pessoas com pouco estudo e em pardos. As lesões autoprovocadas intencionalmente por enforcamento, estrangulamento e sufocação foram mais prevalentes, seguido pelas lesões autoprovocadas intencionalmente por precipitação de um lugar elevado.

Desse modo, torna-se necessário adotar estratégias para que medidas preventivas ao suicídio em idosos sejam implementadas pelos profissionais de saúde para mitigar novas ocorrências. Esta pesquisa contribuiu no sentido de apontar o panorama da mortalidade por suicídio em idosos no Distrito Federal de modo a contribuir para a tomada de decisão para políticas públicas nesta temática devido ao impacto negativo desta problemática. .

\section{REFERÊNCIAS}

1. Barbosa FO, Macedo PCMM, Silveira RMC. Depressão e o suicídio. Rev SBPH [Internet]. 2011 [Acesso em 02 mai 2021];14(1):233-43.

2. Minayo MCS, Cavalcante FG, Mangas RMN, Souza JRA. Autópsias psicológicas sobre suicídio de idosos no Rio de Janeiro. Ciênc Saúde Coletiva [Internet]. 2012 [Acesso em 02 mai 2021]; 17(10):2772-81

3. Organização Panamericana da Saúde. Prevención del suicidio: un imperativo global. Washington, DC: OPS; 2014.

4. BRASIL. Congresso Nacional. Câmara de Deputados. Grupo de trabalho deve analisar políticas de prevenção ao suicídio no Brasil. Brasília (DF); 2016

5. Sousa GS, Silva RMD, Figueiredo AEB, Minayo MCDS, Vieira LJEDS. Circunstâncias que envolvem o suicídio de pessoas idosas. Interface (Botucatu) [Internet]. 2014 [Acesso em $10 \mathrm{abr}$ 2021];18(49):389-402.

6. Cavalcante FG, Minayo MCS, Mangas RMN. Diferentes faces da depressão no suicídio em idosos. Ciênc Saúde Coletiva [Internet]. 2013 [Acesso em 10 abr 2021];18(10):2985-94.

7. World Health Organization. Preventing suicide: A global imperative. Genebra (CH); 2014.

8. United Nations. Department of Economic and Social Affairs. Population Division (2015). World Population Ageing. New York; 2015.

9. Department of Economic and Social Affairs - Population Division. World Population Ageing: 1950-2050. UN Publication; 2002.

10. Brasil. Ministério da Saúde. Portaria n. 1.876, de 14 de agosto de 2006. Institui diretrizes nacionais para prevenção do suicídio. Brasília (DF); 2006.

11. Pinto LW, Pires TO, Silva CMFP, Assis SG. Evolução temporal da mortalidade por suicídio em pessoas com 60 anos ou mais nos estados brasileiros, 1980 a 2009. Ciênc Saúde Coletiva [Internet]. 2012 [Acesso em 10 abr 2021];17(8):1973-81.

12. Seleghim MR, Bellasalma ACM, Mathias TAF, Oliveira MLF. Caracterização das tentativas de suicídio entre idosos. Cogitare Enferm [Internet]. 2012 [Acesso em 10 abr 2021];17(2):277-83.
13. Ciulla L, Nogueira EL, Silva Filho IG, Tres GL, Engroff P, Ciulla V et al. Suicide risk in the elderly: data from Brazilian public health care program. J Affect Disord [Internet]. 2014 [Acesso em 10 abr 2021];152-154:513-6.

14. Minayo MCS,Figueiredo AEB, Mangas RMN. O comportamento suicida de idosos institucionalizados: histórias de vida. Physis [Internet]. 2017 [Acesso em 10 abr 2021];27(4):9811002.

15. Meneghel SN, Gutierrez DMD, Silva RM, Grubits S, Hesler LZ, Ceccon RF. Suicídio de idosos sob a perspectiva de gênero. Ciênc saúde coletiva [Internet]. 2012 [Acesso em 10 abr 2021];17(8):1983-92.

16. Gutierrez DMD, Minayo MCS, Sousa ABL, Grubits S. Pessoas idosas tentam suicídio para chamar atenção? Saúde Soc. São Paulo [Internet]. 2020 [Acesso em 10 abr 2021];29(4):e190659.

17. Gomes AV, Cardoso PKB, Rocha FCV, Carvalho CMS, Sales MCV. Perfil sociodemográfico de idosos vítimas de suicídio em um estado do Nordeste do Brasil. Rev Baiana Enferm [Internet]. 2018 [Acesso em 10 abr 2021];32:e26078.

18. Pinto LW, Assis SG, Pires TO. Mortalidade por suicídio em pessoas com 60 anos ou mais nos municípios brasileiros no período de 1996 a 2007. Ciênc Saúde Coletiva [Internet]. 2012 [Acesso em 10 abr 2021];17(8):1963-72.

19. Rios MA, Anjos KF, Meira SS, Nery AA, Casotti CA. Completude do sistema de informação sobre mortalidade por suicídio em idosos no estado da Bahia. J Bras Psiquiatr [Internet]. 2013 [Acesso em 10 abr 2021];62(2):131-8.

20. Almeida A, Almeida A, Sousa MPL, Liberato LC, Brasil MYO, Silva CRL. O suicídio como um problema de saúde pública. Saúde Coletiva (Barueri) [Internet]. 2021 [Acesso em 25 mai 2021];11(61):5018-22.

21. Aguiar RS. O idoso com déficit de autocuidado em domicílio e as implicações para o cuidador familiar. Rev Enferm UFPE online [Internet]. 2011 [Acesso em 15 mai 2021];5(10):2545-51.

22. Silva EMA, Aguiar RS. Fatores relacionados à polimedicação em idosos e a segurança do paciente: uma revisão integrativa. Nursing (São Paulo) [Internet]. 2020 [Acesso em 15 mai 2021];23(265):4127-33. 